\title{
Infiltration und Grundwasserströmung in geklüftet-porösen Buntsandsteingrundwasserleitern im Osten des Thüringer Beckens
}

\author{
Tino Rödiger · Martin Sauter $\cdot$ Georg Büchel
}

Eingang des Beitrages: 20.5.2008/Eingang des überarbeiteten Beitrages: 7.10.2008/Online veröffentlicht: 9.1.2009

(C) Die Autoren 2009. Dieser Artikel wurde mit Open Access auf Springerlink.com veröffentlicht.

\begin{abstract}
Kurzfassung Im Osten des Thüringer Beckens sind aufgrund der geringen Niederschläge von $585 \mathrm{~mm} / \mathrm{a}$ die Grundwasserressourcen in den geklüfteten Buntsandsteingrundwasserleitern stark limitiert. Ziel der Untersuchung war es, für dieses Buntsandsteinfließsystem ein numerisches Grundwasserströmungsmodell zu erstellen, um die zukünftige Entwicklung der Grundwasserressourcen auf der Grundlage verschiedener Klimaszenarien prognostizieren zu können. Im vorliegenden Beitrag wurden Grundwasserganglinien mit dem Ziel analysiert, eine Inputfunktion für die Grundwasserneubildung am Grundwasserspiegel abzuleiten, welche ein wichtiger Eingangsparameter bei der numerischen Modellierung ist. Es konnte gezeigt werden, dass die Strömung im Buntsandsteingrundwasserleiter sowohl durch ein kluft- sowie ein matrixdominiertes Strömungsregime charakterisiert ist. Der überwiegende Anteil der Grundwasserneubildung infiltriert rasch durch das Kluftsystem. Dagegen bewirken die geringeren Matrixdurchlässigkeiten in der ungesättigten Zone, in Abhängigkeit vom Flurabstand, eine deutliche Verzögerung der Grundwasserneubildung von bis zu mehreren Jahren.
\end{abstract}

Dr. T. Rödiger $(\bowtie)$

Helmholtz-Zentrum für Umweltforschung GmbH - UFZ, Department Hydrogeologie,

Theodor-Lieser Str. 4, 06120 Halle/Saale, Deutschland

E-Mail: tino.roediger@ufz.de

Prof. Dr. M. Sauter

Geowissenschaftliches Zentrum der Universität Göttingen,

Abt. Angewandte Geologie,

Goldschmidtstrasse 3, 37077 Göttingen, Deutschland

E-Mail: martin.sauter@geo.uni-goettingen.de

Prof. Dr. G. Büchel

Friedrich-Schiller Universität, Institut für Geowissenschaften,

Burgweg 11, 07749 Jena, Deutschland

E-Mail: georg.buechel@uni-jena.de
Infiltration and groundwater flow into a fractured porous sandstone aquifer in the eastern Thuringian Basin

\begin{abstract}
In the eastern Thuringian Basin, the low precipitation rate of $585 \mathrm{~mm} / \mathrm{y}$ limits the groundwater resources in the sandstone aquifer system. The aim of this study was to develop a groundwater flow model for the region in order to forecast the impacts of different climate scenarios on the groundwater balance. Since recharge is of high relevance for the numerical model, a time series of groundwater levels were investigated to obtain the recharge input function. In the sandstone aquifer it could be shown that groundwater flow is characterised by flow in the fracture network as well as in the matrix. The largest fraction of recharge reaches the water table very quickly via the fracture network. However, both the thickness of the unsaturated zone and the low hydraulic conductivity of the sandstone matrix control the time lag of groundwater recharge. The time lag of the slow component to reach the water table is in the order of several years.
\end{abstract}

Keywords groundwater level - fractured porous sandstone aquifer · water table fluctuation ' groundwater recharge geostatistical estimation

\section{Einleitung}

Im Osten des Freistaats Thüringen wird der Untere und Mittlere Buntsandstein intensiv als Grundwasserleiter für die Trinkwasserversorgung zahlreicher Städte genutzt (Hecht 1995). Das $791 \mathrm{~km}^{2}$ große Untersuchungsgebiet erstreckt sich in Ostthüringen südlich und östlich der Stadt Jena und ist Teil des oberirdischen Einzugsgebiets der Saale (Abb. 1). Im Untersuchungsgebiet streicht der Buntsandstein auf ei- 


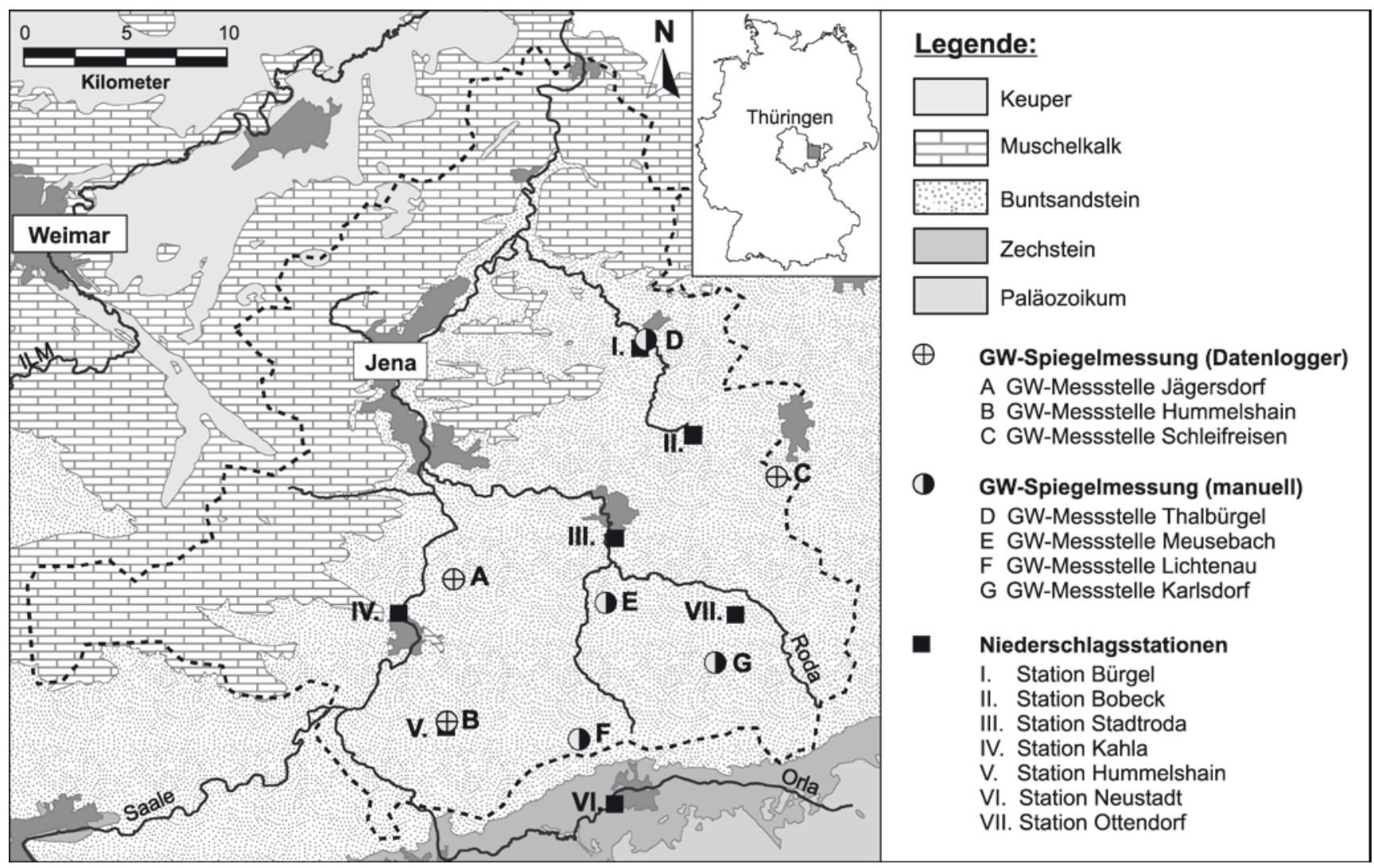

Abb. 1 Lage (gestrichelt) und geologischer Aufbau des Untersuchungsgebietes. Die Buchstaben A bis G markieren Grundwassermessstellen (GW-Messstellen) und die Zahlen I. bis VII. lokalisieren Niederschlagsstationen

ner Fläche von ca. $513 \mathrm{~km}^{2}$ aus. Die Bernburg-Folge (oberer Abschnitt des Unteren Buntsandsteins) und der gesamte Mittlere Buntsandstein werden als einheitlicher Grundwasserleiter suB/sm für die Trinkwassergewinnung der Region genutzt. Im Arbeitsgebiet liegen im geklüfteten Festgesteinsgrundwasserleiter überwiegend ungespannte Verhältnisse vor. Nur lokal führen Faziesvariationen zu gespannten Verhältnissen. Mit der Überdeckung des Grundwasserleiters durch den Hangendstauer des Oberen Buntsandsteins tritt ein Wechsel von ungespannten zu gespannten Verhältnissen ein. Die Grundwasserströmung ist entscheidend durch das $2^{\circ}-5^{\circ} \mathrm{N}$ bzw. NW gerichtete Einfallen der Schichten in Richtung Zentrum des Thüringer Beckens geprägt.

Das Neubildungsgebiet zählt mit ca. $585 \mathrm{~mm} / \mathrm{a}$ zu den niederschlagärmsten Regionen Thüringens. Im Zuge einer nachhaltigen Ressourcenbewirtschaftung wurde für das Buntsandsteinfließsystem ein numerisches Grundwasserströmungsmodell erstellt. Aus diesem Grund wurden im vorliegenden Beitrag Grundwasserganglinien mit dem Ziel analysiert, eine Inputfunktion für die Grundwasserneubildung am Grundwasserspiegel abzuleiten. Dazu wurden statistische Korrelationsverfahren zur Bestimmung der zeitlichen Verzögerung zwischen Niederschlagsereignis und der Reaktion am Grundwasserspiegel verwendet. Anschließend erfolgte durch die Kombination der WTF (water table fluctuation)Methode (Healy \& Cook 2002) mit einem Bodenwasserhaushaltsmodell (Sauter 1992) eine Volumenabschätzung der dualen Fließsysteme. Eine vergleichbare Verknüpfung der Methoden zur Quantifizierung der Grundwasserneubildung wurde von Sophocleous (1991) gewählt. Ziel der Untersuchungen war es, den Effekt von kluftdominierter und matrixdominierter Strömung, sowohl in der gesättigten als auch ungesättigten Zone, sowie die Auswirkung unterschiedlicher Grundwasserflurabstände auf die Grundwasserströmung zu quantifizieren. Der Effekt des dualen Fließverhaltens wurde bereits in anderen deutschen Buntsandsteinregionen festgestellt (Hauthal 1967, Matthess 1970, Udluft 1971, März 1977).

\section{Material und Methoden}

Durch die Korrelationen von Grundwasserständen mit Niederschlagsereignissen sind Aussagen über die Grundwasserdynamik im Grundwasserleiter suB/sm möglich. Durch die Konstruktion von Mittelsenkrechten auf den Verbindungs- 
Tabelle 1 Grundwasserspiegelaufzeichnungen (Thüringer Landesanstalt für Umwelt und Geologie)

\begin{tabular}{lccc}
\hline $\begin{array}{l}\text { Automatisiert } \\
\text { (Datenlogger) }\end{array}$ & $\begin{array}{c}\text { Flurabstand } \\
\text { [m u. GOK] }\end{array}$ & $\begin{array}{c}\text { Messzeitraum } \\
\text { (Grundwasserspiegel) }\end{array}$ & Datenlücken \\
\hline Schleifreisen & $\sim 16$ & $27.03 .2000-10.10 .2007$ & $09.04 .2001-23.07 .2001$ \\
Jägersdorf & $\sim 35$ & $05.03 .1997-29.04 .2004$ & $24.02 .2003-10.09 .2003$ \\
Hummelshain & $\sim 62$ & $07.03 .1994-29.04 .2004$ & $23.03 .1994-18.08 .1994$ \\
& & & $14.11 .1994-23.02 .1995$ \\
& & & $28.10 .1998-28.05 .2001$ \\
& & & $21.08 .2001-07.08 .2002$ \\
Manuell & Flurabstand & Messzeitraum & \\
Karlsdorf & {$[\mathrm{m}$ u. GOK] } & $($ Grundwasserspiegel) & Datenlücken \\
Thalbürgel & $\sim 7$ & $08.05 .1973-22.06 .2003$ & \\
Meusebach & $\sim 1$ & $26.11 .1964-22.05 .2005$ & $16.10 .1975-30.09 .1991$ \\
Lichtenau & $\sim 6$ & $01.05 .1973-22.06 .2003$ & \\
\hline
\end{tabular}

linien der Niederschlagsstationen wurden Thiessen-Polygone erstellt (Hölting 1992), denen die Grundwassermessstellen zugeordnet wurden. Das System ist charakterisiert durch die Analyse langjähriger Grundwasserspiegelganglinien von sieben Grundwassermessstellen (Tabelle 1). Die Auswahl der Messstellen erfolgte auf der Basis der unterschiedlichen Flurabstände und der Option, sie dem Grundwasserneubildungs-, Transit- und Entlastungsgebiet zuordnen zu können. Alle Messstellen sind im Grundwasserleiter suB/sm ausgebaut. Die Grundwasserspiegelaufzeichnungen wurden von der Thüringer Landesanstalt für Umwelt und Geologie (TLUG) zur Verfügung gestellt. Für die Grundwassermessstellen Hummelshain, Jägersdorf und Schleifreisen (Abb. 1) existieren seit den Jahren 1994, 1997 und 2000 mehr oder weniger kontinuierliche Aufzeichnungen aus Datenloggern (Messintervall ca. 6 Stunden). Für die übrigen Messstellen gibt es manuelle Wasserspiegelaufzeichnungen von teilweise mehr als 30 Jahren (Messinterval ca. 7 Tage).

Die Korrelationsergebnisse zwischen Niederschlag und Grundwasserstand wurden zur Generierung von Inputfunktionen für die Grundwasserneubildung am Grundwasserspiegel in Abhängigkeit des Flurabstandes in einem numerischen Strömungsmodell eingesetzt (Rödiger 2005). Die Berechnungen des Modells erfolgten mit der Software VISUAL MODFLOW 3.0 (WHI 2002).

\section{Korrelationsverfahren}

Zusammenhänge zwischen rasch reagierenden Fließsystemen und Niederschlagsmengen können durch die Methoden der Zeitreihenzerlegung und der Kreuzkorrelation (Davis 1986, Merkel \& Planer-Friedrich 2002) analysiert werden. Dagegen lassen sich Beziehungen zwischen langsam zirkulierenden Fließsystemen und Niederschlagsreihen durch die
Kombination der Verfahren des gleitenden Durchschnitts und der Korrelation wiedergeben. Für die Korrelationsverfahren wurden die gemessenen Niederschlagsmengen [mm/d] verwendet. Die berechneten Tageswerte für die Grundwasserneubildung aus der Bodenwasserbilanzberechnung (Sauter 1992) wurden für die Korrelationen nicht berücksichtigt, da sie von der Parametrisierung einzelner Prozesse bei der Bodenwasserbilanzberechnung abhängen und so Unschärfen auftreten können.

Für die zeitliche Analyse eines rasch reagierenden Fließsystems (Tage) sind für das Verfahren der Kreuzkorrelation trendbereinigte Grundwasserganglinien nötig, in denen Zyklen, Ausreißer und saisonale Effekte eliminiert werden. Aus diesem Grund wurde für die Grundwasserganglinien der Datenloggeraufzeichnungen die Methode einer Zeitreihenzerlegung angewendet. Hierbei wurde die Zeitreihe in zeitlich kleinere Segmente von jeweils ein bis drei Monaten unterteilt. Jeder Datenwert eines Segmentes wurde durch den entsprechenden Mittelwert dividiert.

Die anschließende Kreuzkorrelation c zeigt, inwieweit eine zeitliche Verschiebung (time-lag $=\tau$ ) zwischen den Niederschlägen $x\left(t_{i}\right)$ und den Grundwasserständen $y\left(t_{i}\right)$ besteht. Damit kann die Verzögerung der schnellen Grundwasserneubildung innerhalb eines Kluftsystems abgeschätzt werden (Gl. 1).

[1] $\quad c_{x y}\left(\tau \tau=\int_{-\infty}^{\infty} x(t+\tau) y(t) d t\right.$

Es werden hierbei keine prozessorientierten, sondern ausschließlich statistische Zusammenhänge betrachtet, deren $\mathrm{Maß}$ mittels der Kovarianz COV (Gl. 2) beschrieben werden kann.

[2] $\operatorname{COV}=\sum_{i=1}^{n} \frac{\left(x_{i}-\bar{x}\right)\left(y_{i}-\bar{y}\right)}{n-1}$ 
Die Zeitreihen $\mathrm{x}$ und y müssen ein äquidistantes Messintervall besitzen. Die Symbole $\bar{x}$ und $\bar{y}$ sind das arithmetische Mittel und $\mathrm{n}$ bezeichnet die Anzahl der Werte in den Zeitreihen $\mathrm{x}_{\mathrm{i}}$ und $\mathrm{y}_{\mathrm{i}}$. Für verschiedene Versatzbeträge $\tau$ wird ein Korrelationskoeffizient $r$ aus dem Verhältnis der Kovarianz COV der Zeitreihen $\mathrm{x}\left(\mathrm{t}_{\mathrm{i}}\right)$ und $\mathrm{y}\left(\mathrm{t}_{\mathrm{i}}\right)$ und dem Produkt der Standardabweichungen $\mathrm{S}_{\mathrm{x}}$ und $\mathrm{S}_{\mathrm{y}}$ berechnet (Gl. 3). Der Korrelationskoeffizient $r$ ist ein dimensionsloses Maß für den Grad der Korrelation und zeigt, bei welcher zeitlichen Verschiebung $\tau$ die größte Übereinstimmung zwischen den zwei Zeitreihen zu beobachten ist.

[3] $\mathrm{r}=\frac{\mathrm{COV}}{\mathrm{S}_{\mathrm{x}} \cdot \mathrm{S}_{\mathrm{y}}}$

Die Methode des gleitenden Durchschnitts wurde auf die langjährigen Niederschlagszeitreihen im Zeitraum 1990-2002 angewendet, um die zeitliche Verzögerung der langsamen Grundwasserneubildungskomponente im Matrixsystem abschätzen zu können.

Durch dieses Verfahren werden zufällige Einzelschwankungen in den Niederschlagszeitreihen nicht berücksichtigt. In Abhängigkeit des Grades der Glättung werden saisonale Effekte verstärkt oder nicht berücksichtigt. Die Unterschiede zwischen expliziten Nass- und Trockenjahren bleiben jedoch erhalten. Der Grad der Glättung ist abhängig von der Anzahl benachbarter Punktem, die zur Bildung des jeweiligen Mittels verwendet werden (Gl. 4). Es wurden 365 Glättungspunkte verwendet, was dem gleitenden Durchschnitt mit einem Fenster von einem Jahr entspricht.

$$
\begin{aligned}
x_{t}= & \frac{1}{2 m+1}+\left(x_{t-m}+x_{t-m+1}+\ldots+x_{t-1}+x_{t}+\right. \\
& \left.x_{t+1}+\ldots x_{t+m}=\right)
\end{aligned}
$$

Im Anschluss wurde die zeitliche Verteilung der Nassund Trockenjahre mit den gemessenen Grundwasserständen korreliert.

\section{WTF-Methode nach Healy \& Cook (2002)}

Die Grundwasserneubildungsanteile der kluft- und matrixdominierten Strömungsregime im Buntsandstein wurden mithilfe der WTF (,water table fluctuation“)-Methode nach Healy \& Cook (2002) quantifiziert. Die Methode basiert auf der Annahme, dass der Grundwasserspiegel mit nur kurzer Verzögerung auf Niederschlagsimpulse reagiert bzw. dass die Änderungen am Grundwasserspiegel überwiegend durch die resultierende Grundwasserneubildung verursacht werden. Aus diesem Grund ist diese Methode in Gebieten mit geringem Flurabstand indiziert. Trotz der vorhandenen großen Flurabstände im Untersuchungsgebiet, wurde das Verfahren eingesetzt, da die Grundwasserspiegelaufzeichnungen in den Messstellen Hummelshain, Jägersdorf und
Schleifreisen die erforderlichen schnellen Anstiege und das anschließend rasche Abfallen der Grundwasserstände zeigten. Nach der WTF-Methode berechnet sich der Anteil der schnellen Komponente der Grundwasserneubildung R [L/T] aus dem Speicherkoeffizienten S [-] und der Änderung der Grundwasserspiegelhöhe $\Delta \mathrm{h}[\mathrm{L}]$ im entsprechenden Zeitraum $\Delta \mathrm{t}[\mathrm{T}]$ (Gl. 5). Die WTF-Methode hängt dabei stark vom Speicherkoeffizienten ab, da dieser die unsicherste zu bestimmende Größe und somit die größte Fehlerquelle darstellt.

[5] $\mathrm{R}=\mathrm{S} \cdot \frac{\Delta \mathrm{t}}{\Delta \mathrm{t}}$

Zur Berechnung der einzelnen Grundwasserneubildungsanteile im Grundwasserleiter suB/sm wurde zunächst der Speicherkoeffizient $S$ ermittelt (Gl. 6). Dieser ergibt sich aus dem Zusammenhang, dass im ungespannten Grundwasserleiter durch Grundwasserabfluss der Porenraum $V_{P}$ entleert wird. Diese Wassermenge entspricht dem unterirdischen Abfluss $A_{U}\left[L^{3}\right]$, der für Oberflächenabfluss korrigiert, über einen Zeitraum $\Delta t$, an einem Vorfluterpegel bestimmt wurde. Das Gesamtaquifervolumen $\mathrm{V}_{\mathrm{G}}\left[\mathrm{L}^{3}\right]$, in welchem die Entwässerung stattfindet, ist das Produkt der Änderung der Wasserspiegelhöhe $\Delta \mathrm{h}$ während des Zeitraums $\Delta \mathrm{t}$ und der Fläche des dazu gehörigen Einzugsgebietes $F\left[\mathrm{~L}^{2}\right]$.

$$
\mathrm{S}=\frac{\mathrm{A}_{\mathrm{U}}}{(\Delta \mathrm{h} \cdot \mathrm{F})}=\frac{\mathrm{V}_{\mathrm{P}}}{\mathrm{V}_{\mathrm{G}}}
$$

\section{Ergebnisse und Diskussion}

Interpretation der Grundwasserganglinien

Die Datengrundlage zur Auswertung der Hydrodynamik im Buntsandsteinfließsystem bilden die in den Abb. 2 und 3 dargestellten Grundwasserganglinien, die nach Flurabständen unterteilt wurden. Aufgrund der räumlichen Distanz zwischen Messstellen und Trinkwasserförderanlagen ist eine Beeinflussung der beobachteten Grundwasserstände durch die Trinkwasserförderung nicht zu erwarten. Ebenfalls ist aufgrund der vorherrschenden effluenten Verhältnisse im Untersuchungsgebiet und durch die größere Entfernung der Messstellen zu den Vorflutern ein influenter Zustrom auszuschließen.

Die Grundwasserganglinien der Messstellen mit Flurabständen von $<10 \mathrm{~m}$ zeigen deutliche saisonale Variationen mit ausgeprägten Minima und Maxima (Abb. 2). Es ist zu erkennen, dass die Wasserspiegel unmittelbar bzw. um wenige Tage verzögert auf Niederschlagsereignisse reagieren. Die schnellen Reaktionen auf Niederschläge sind überwiegend auf die geringen Flurabstände und die damit verbundenen kurzen Infiltrationswege zurückzu- 
Abb. 2 Manuelle Grundwasserspiegelaufzeichnungen (schwarze Linien - Flurabstand (GW-Spiegel unter Geländeoberkante GOK)) der Messstellen Karlsdorf, Lichtenau, Meusebach und Thalbürgel sowie Niederschlagshöhen

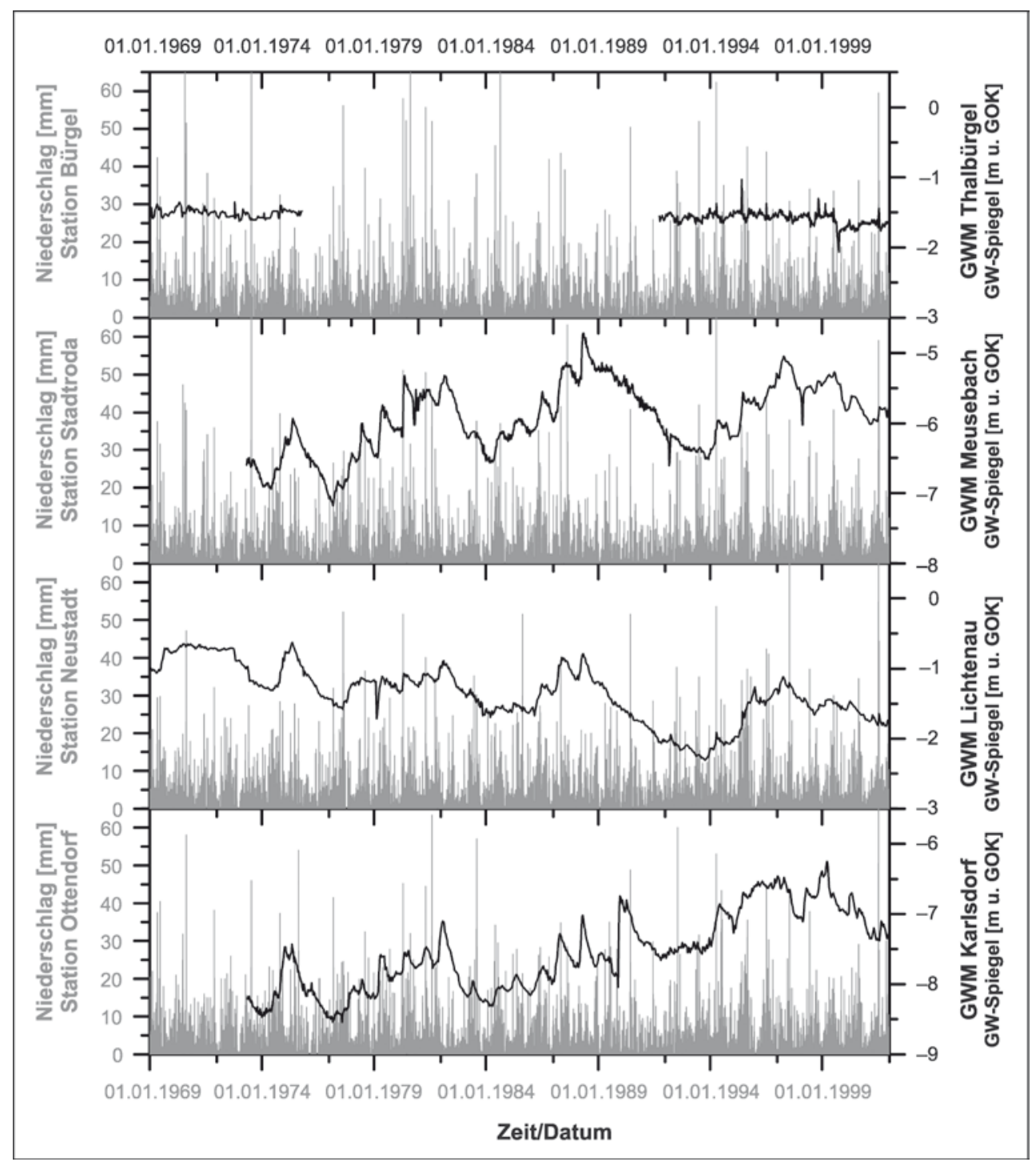

führen. Eine zeitliche Einordnung von $<8$ Tagen kann für die Reaktion des Grundwasserspiegels auf Niederschlagsereignisse in diesen Messstellen nicht aufgelöst werden, da die Wasserspiegelaufzeichnungen nur eine Auflösung von 7 Tagen besitzen.

In Abb. 3 sind die hoch aufgelösten Grundwasserganglinien von Messstellen mit einem Flurabstand von $>10 \mathrm{~m}$ dargestellt. Es ist zu erkennen, dass die Messstellen Hummelshain, Jägersdorf und Schleifreisen ausgeglichene Grundwasserganglinien zeigen, die keine saisonalen Minima und Maxima aufweisen. Weiterhin ist zu beobachten, dass die Ganglinien sowohl kurzfristige Schwankungen (im Weiteren als Peaks bezeichnet) und langfristige (mehrjährige) Schwankungen der Wasserstände aufweisen. Dieses gleichzeitige Auftreten von Peaks und mehrjährigen Schwankungen wird auf zeitlich voneinander unabhängige Faktoren, die den Grundwasserspiegel beeinflussen, sowie auf das deutlich ausgebildete duale Fließverhalten im Buntsandstein zurückgeführt.

Die scharfe Geometrie der Peaks mit raschem Anstieg und Abfall im Zentimeter- bis Dezimeterbereich zeigt eine rasche Reaktion des Grundwasserspiegels. Diese ist bedingt durch ein schnelles Fließsystem, wie z. B. ein Kluftsystem. Trotz der großen räumlichen Distanz von ca. $7 \mathrm{~km}$ zwischen den Messstellen Jägersdorf und Hummelshain sowie von ca. $20 \mathrm{~km}$ zwischen diesen und der Messstelle Schleifreisen, treten einzelne Peakmuster zeitgleich in allen drei Ganglinien auf. Daraus ist abzuleiten, dass der Grundwasserleiter relativ einheitlich auf Niederschlags- bzw. Grundwasserneubildungsereignisse reagiert. Trotz der relativ scharfen Geometrie der Peaks ist in Abb. 3 jedoch keine direkte Korrelation zwischen den Peaks und dem Niederschlagsereignis (N) zu erkennen. In Abhängigkeit von der Infiltrationshöhe und -geschwindigkeit sowie der Länge des Fließweges durch 
Abb. 3 Automatische Grundwasserspiegelaufzeichnungen durch Datenlogger (schwarze Linien - Flurabstand (GW-Spiegel unter Geländeoberkante GOK))in den Grundwassermessstellen (GWM) Schleifreisen, Hummelshain und Jägersdorf im Vergleich mit den Niederschlagsereignissen (graue Säulen) der entsprechenden Niederschlagsstationen

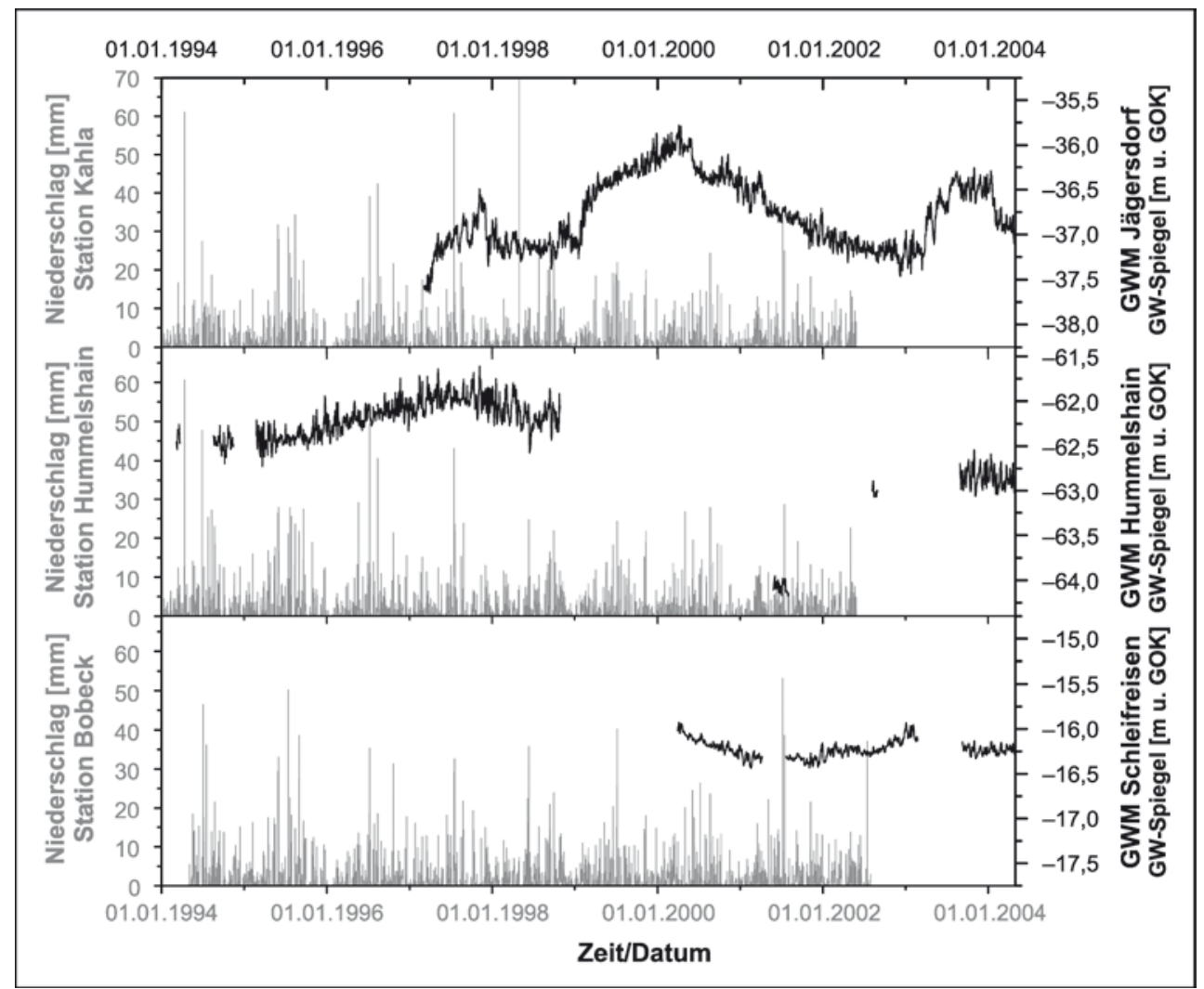

die ungesättigte Zone wird die Grundwasserneubildung abgeschwächt, und die Reaktion des Grundwasserspiegels erfolgt zeitlich verzögert. Hinzu kommt, dass nicht sämtliche Niederschlagsereignisse zur Grundwasserneubildung und damit zur Infiltration führen. So bewirkt die oberste ausgetrocknete Bodenschicht besonders in den Sommermonaten einen erhöhten Oberflächenabfluss, was sich negativ auf die Infiltration auswirkt.

Im Gegensatz zu den Peaks deuten die langfristigen Schwankungen, die sich über Zeiträume von mehreren Jahren erstrecken können und Amplituden von einigen Dezimetern bis maximal 1 Meter aufweisen, auf ein langsames bzw. träge reagierendes matrixdominiertes Fließsystem hin. Die langjährigen Schwankungen der Grundwasserganglinien unterscheiden sich hinsichtlich deren zeitlichen Verlaufs und der beobachteten Amplituden nur geringfügig (Abb. 3). Aus den mitunter mehrjährigen Perioden wird deutlich, dass es sich nicht um saisonale Effekte des Sommer- und Winterhalbjahres handelt.

Analyse der Grundwasserganglinien

Wie im vorigen Abschnitt beschrieben, kann anhand der Aufzeichnungen der Grundwasserganglinien auf ein schnell und ein langsam zirkulierendes Fließsystem geschlossen werden. Zusammenhänge zwischen dem schnell zirkulie- renden Fließsystem und den Niederschlagsmengen wurden durch die Kombination der Methoden der Zeitreihenzerlegung und der Kreuzkorrelation analysiert. Für die Beziehung zwischen dem langsam zirkulierenden Fließsystem und den langjährigen Niederschlagsreihen wurden die Verfahren des gleitenden Durchschnitts und der Korrelation angewendet. Mit den im Abschnitt „Material und Methoden“ vorgestellten statistischen Verfahren wurde geprüft, inwieweit eine zeitliche Verschiebung zwischen den Niederschlagszeitreihen und den verschiedenen Schwankungsperioden der hoch aufgelösten Grundwasserstände besteht. Hierfür sollten die Zeitreihen in einem äquidistanten Messintervall vorliegen. Aus diesem Grund wurden aus den hochauflösenden Grundwasserganglinien (Messintervall $6 \mathrm{~h}$ ) mithilfe des arithmetischen Mittels Tageswerte berechnet. Die anschließende Korrelation über den gesamten Zeitraum der Datenloggeraufzeichnungen ohne vorherige Trend- bzw. Ereignisbereinigung war nicht signifikant. Gründe sind in der starken Variabilität der verschiedenen Grundwasserneubildungsereignisse (zeitlich, räumlich, Intensität) und in den Unterschieden der präferenziellen Fließwege und des Flurabstandes zu suchen. Eine Trend- bzw. Ereignisbereinigung wurde mittels Zeitreihenzerlegung durchgeführt. Erst durch eine weitere Unterteilung der bereinigten Grundwasserganglinienzeitreihe in kleinere Zeitintervalle von jeweils ein bis drei Monaten war es möglich, signifikante Kreuzkorrelationen 
durchzuführen, aus denen eine zeitliche Verschiebung zwischen Niederschlagsereignis und zugehöriger Reaktion des Grundwasserspiegels abzuleiten war.

In Abb. 4 sind die zeitlichen Verschiebungen (,timelag“ $\tau$ ) zwischen Peaks und gemessenen Niederschlagsereignissen und deren Häufigkeiten für die verschiedenen Zeitintervalle ( 1 bis 3 Monate) dargestellt. Als Kriterien für die zeitlichen Verschiebungen (,time-lag“ $\tau$ ) wurden n/2 (Hälfte der Zeitreihenwerte) und Korrelationskoeffizienten von $r>0,7$ für hohe Korrelationen verwendet. Die ermittelten zeitlichen Verschiebungen (,time-lag“ $\tau$ ) für das Zeitintervall von einem Monat wurden mit den Ergebnissen für das Zeitintervall von 2 bzw. 3 Monaten verglichen. Dabei ergaben sich überwiegend die gleichen zeitlichen Verschiebungen (,time-lag“ $\tau$ ) bis zu einem maximalen Versatz von 15 Tagen. Ab einem ,time-lag“ $\tau>15$ konnten in den Zeitintervallen von 2 bzw. 3 Monaten keine signifikanten Verzögerungen $\tau$ berechnet werden. Die Ergebnisse $\tau<15$ spiegeln trotz der großen Variabilität die Reaktion eines schnell reagierenden Fließsystems wieder. Die Variabilität ist dabei von verschiedenen Faktoren während des Grundwasserneubildungsprozesses (z. B. Infiltrationshöhe und -geschwindigkeit) abhängig. Für die Messstellen Jägersdorf und Hummelshain mit Flurabständen $>30 \mathrm{~m}$ ergaben sich korrelierte Versätze von bis zu 14 Tagen. Dagegen wurde für die Messstelle Schleifreisen, mit einem Flurabstand von ca. $15 \mathrm{~m}$, ein Versatz von bis zu 6 Tagen ermittelt. In allen drei Messstellen lassen sich die geringen zeitlichen Versätze von wenigen Tagen, zwischen dem Niederschlagsereignis und der entsprechenden Reaktion des Grundwasserspiegels, durch die gute Klüftigkeit und die damit verbundenen hohen Infiltrationsraten im Buntsandsteinaquifer erklären. Das infiltrierende Sickerwasser bewegt sich vorwiegend in Kluftzonen und wird dem Grundwasserleiter rasch zugeführt.

Zur Berechnung der Reaktionszeiten des langjährigen Matrixfließsystems wurden die gemessenen mehrjährigen Wasserstandsänderungen dem Auftreten von Nass- und Trockenjahren (Abb. 5) gegenübergestellt. Letztere wurden aus dem gleitenden Durchschnitt langjähriger Niederschlagszeitreihen ermittelt. Anhand des deutlich ausgebildeten Minimums in Abb. 5 ist zu erkennen, dass 1991 ein signifikantes Trockenjahr war. Dagegen lassen sich der maximale Anstieg von 1993 und die gut ausgebildeten Maxima 1994 und 1995 auf Nassjahre zurückführen.

Den drei Datenloggerstandorten Jägersdorf, Schleifreisen und Hummelshain wurden den Thiessen-Polygonen entsprechend die Niederschlagsstationen Kahla, Bobeck und Hummelshain zugeordnet. Aus der Beziehung zwischen geglätteter Niederschlagsganglinie der Station Kahla und den langfristigen Schwankungen des Grundwasserganges in der Messstelle Jägersdorf wurde eine signifikante zeitliche Verschiebung von ca. 5 1/2 Jahren abgeleitet (Abb. 6).

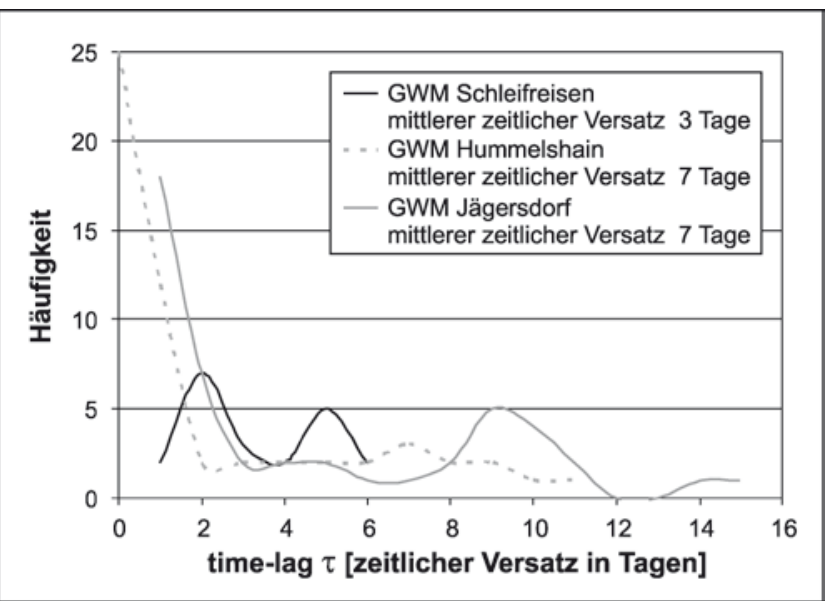

Abb. 4 Darstellung der Häufigkeiten der signifikanten Versatzbeträge (time-lag $\tau$ ), die sich aus der Korrelationen zwischen den Peaks der GW-Spiegelaufzeichnungen der Datenlogger und den gemessenen Niederschlagsereignissen der entsprechenden Niederschlagsstationen ergeben. Aus der Variationsbreite des time-lags $\tau$ wurde für jede Messstelle ein mittlerer zeitlicher Versatz (Fließzeit) berechnet

Dagegen erschweren die kurzen und nicht kontinuierlichen Wasserspiegelaufzeichnungen in der Messstelle Schleifreisen Aussagen, sodass sich keine eindeutigen Korrelationen ableiten lassen. In Abb. 7 ist das wahrscheinlichste Korrelationsergebnis mit einer zeitlichen Differenz von ca. 5 Jahren für die Messstelle Schleifreisen dargestellt. Im Bereich der Messstelle Hummelshain ist eine plausible Auswertung aufgrund der großen Datenlücken in den Aufzeichnungen nicht möglich. Geht man von einer direkten Proportionalität zwischen Flurabstand und Laufzeitdifferenz aus, müsste die Messstelle Hummelshain mit einem Flurabstand von über $60 \mathrm{~m}$ eine größere Laufzeitdifferenz zwischen Niederschlag und der mehrjährigen Schwankung aufweisen. Unter dieser Annahme zeigt die Abb. 8 die bestmögliche Anpassung. Die zeitliche Verschiebung zwischen dem Niederschlagsmaximum und dem langfristigen Grundwasserstandsmaximum am Standort Hummelshain beträgt demnach ca. 10 Jahre. Grund für die zunehmend verzögerten Reaktionen der Grundwasserneubildung ist die Verlängerung der Infiltrationswege in der feinklüftigen Matrix des ungesättigten Buntsandsteins, ein Zusammenhang der auch in anderen deutschen Buntsandsteinaquiferen zu beobachten ist (Harzer 2003). Unter Annahme einer gravitativen Entleerung lassen sich Sickergeschwindigkeiten für das Kluft- und Porensystem aus den verschiedenen Verweilzeiten in Abhängigkeit der Flurabstände bilanzieren. Multipliziert man diese im Anschluss mit einem Speicherkoeffizient von $3 \%$ für das Kluftsystem aus Gleichung 4 und $5 \%$ für das Porensystem (siehe Abschnitt „Quantifizierung der Grundwasserneubildungsanteile mittels WTF-Methode"), ergeben sich spezifische Durchflüsse von $1 \cdot 10^{-6}$ bis $7 \cdot 10^{-7} \mathrm{~m} / \mathrm{s}$ für das Kluftsystem 
Abb. 5 Mittels der Methode des gleitenden Durchschnitts abgeleitete Niederschlagsglättungskurven von verschiedenen Niederschlagsstationen im Untersuchungsgebiet. Zu erkennen ist deutlich die Verteilung der Nass- und Trockenjahre

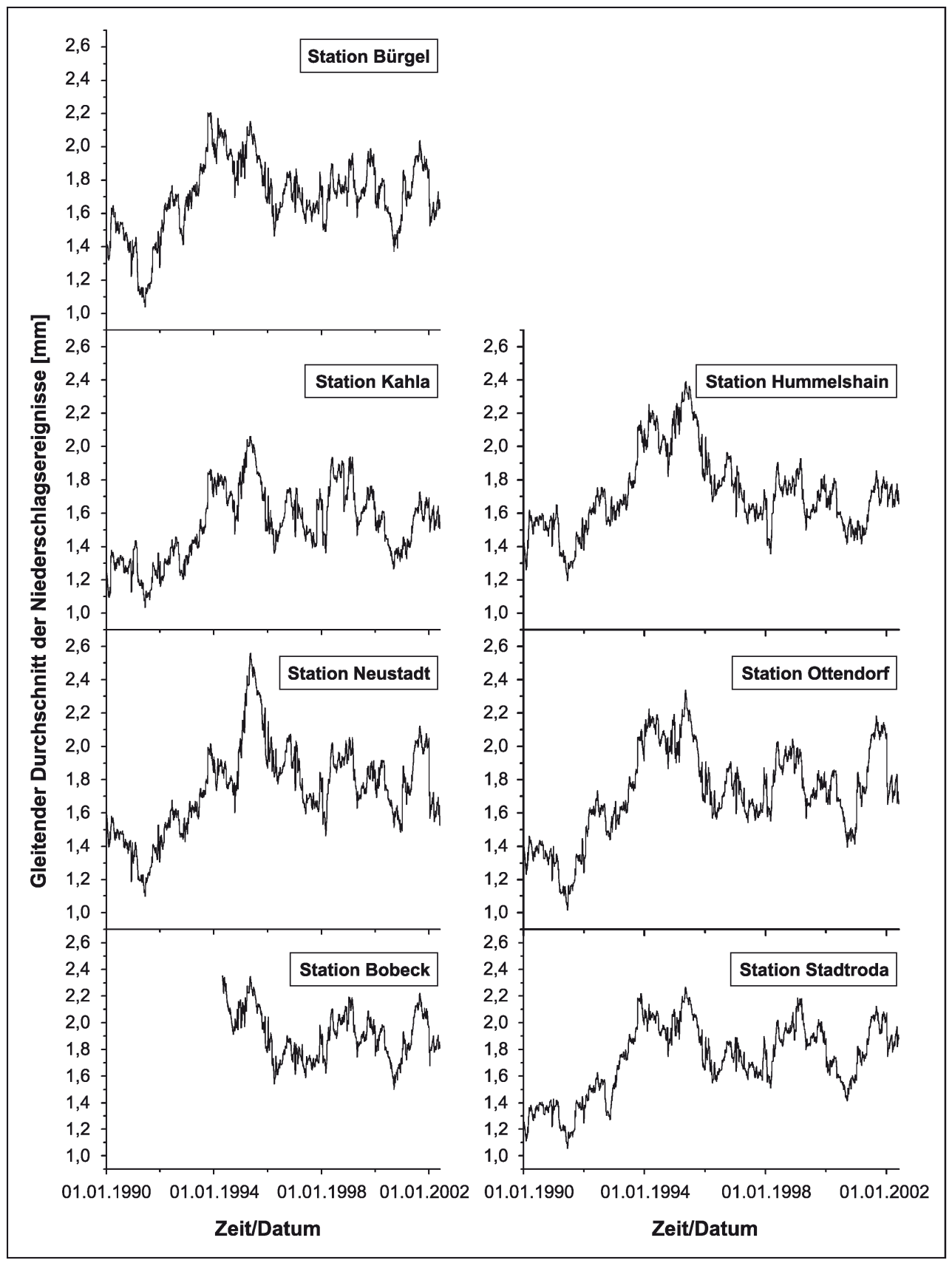

und von $5 \cdot 10^{-9}$ bis $9 \cdot 10^{-9} \mathrm{~m} / \mathrm{s}$ für das Porensystem, welche unter den gegebenen Annahmen dem Durchlässigkeitsbeiwert $\mathrm{k}_{\mathrm{f}}$ entsprechen. Die berechneten Werte und Größenunterschiede für Kluft- und Porensysteme stimmen gut mit den Literaturangaben in anderen deutschen Sandsteingebieten überein. Im Unteren und Mittleren Buntsandstein haben Matthess (1970), Udluft (1971) und März (1977) für die Gesteinsdurchlässigkeit $\mathrm{k}_{\mathrm{f}}$-Werte zwischen $10^{-11}$ und $10^{-7} \mathrm{~m} / \mathrm{s}$ bestimmt, während nach Hauthal (1967), Dürbaum et al. (1969), Einsele \& Merklein (1978), Georgotas \& Udluft (1978) und Schraft \& Rambow (1984) für die Gebirgsdurchlässigkeit $\mathrm{k}_{\mathrm{f}}$-Werte von $10^{-7}$ bis $10^{-3} \mathrm{~m} / \mathrm{s}$ errechnet wurden.
Im Bereich der Merseburger Buntsandsteinplatte wurden durch Hauthal (1967) ebenfalls unterschiedliche Infiltrationsgeschwindigkeiten für Großklüfte und die feinklüftige Matrix festgestellt. Innerhalb der Großklüfte lag der $\mathrm{k}_{\mathrm{f}}$-Wert mit ca. $10^{-6} \mathrm{~m} / \mathrm{s}$ um den Faktor 20 bis 30 höher als in dem feinklüftigen Matrixsystem.

Quantifizierung der Grundwasserneubildungsanteile mittels WTF-Methode

Zur Berechnung der Grundwasserneubildungsanteile mittels der WTF-Methode sind Speicherkoeffizienten für das Bunt- 
Abb. 6 Korrelation zwischen geglätteter Niederschlagsganglinie der Station Kahla und den langfristigen Schwankungen des Grundwasserganges in der Messstelle Jägersdorf
Abb. 7 Wahrscheinlichste Korrelation zwischen geglätteter Niederschlagsganglinie der Station Bobeck und den langfristigen Schwankungen des Grundwasserganges in der Messstelle Schleifreisen
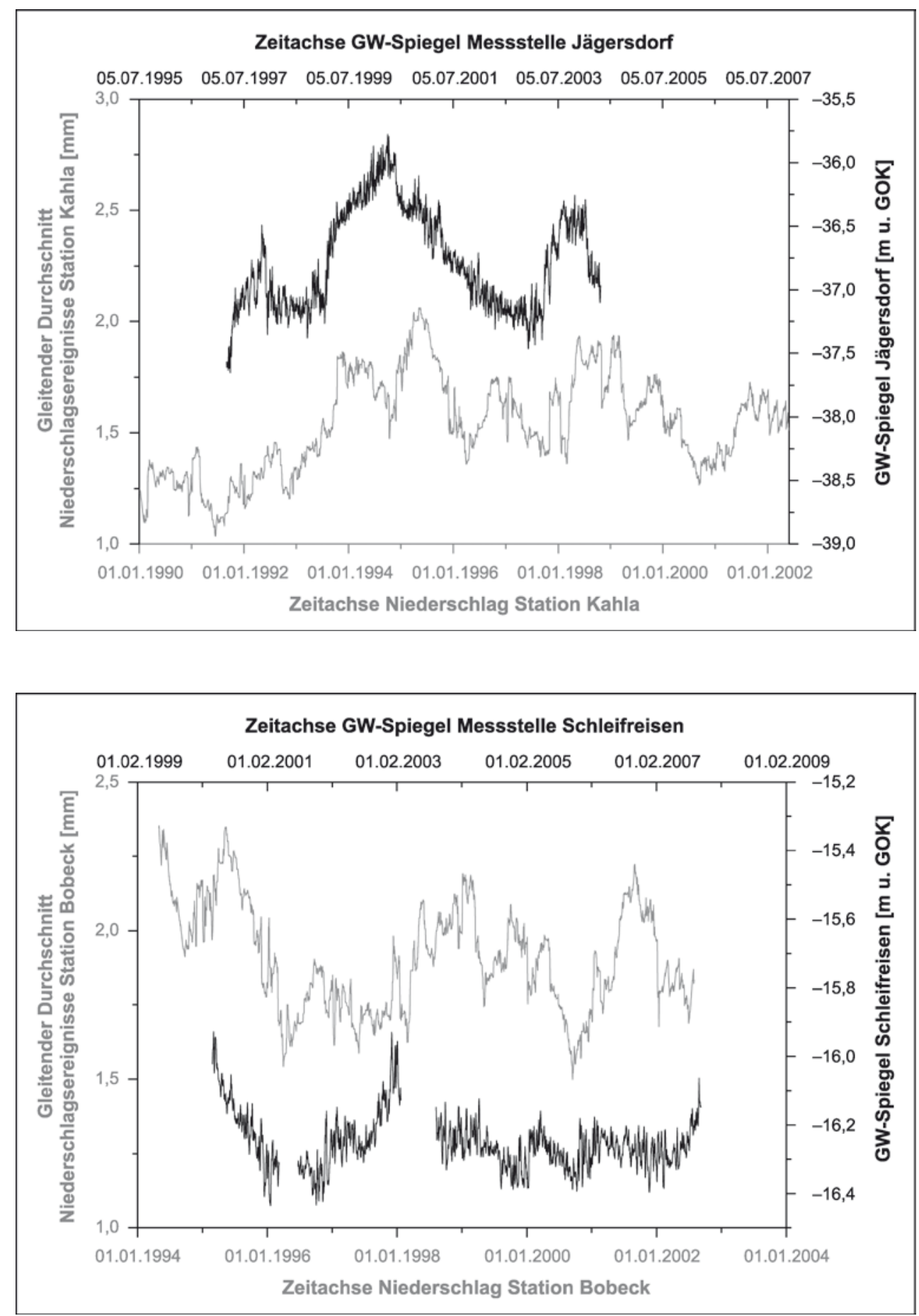

sandsteinsystem notwendig. Hierfür wurden im Zeitraum 1994 bis 2001 die detaillierten Datenloggeraufzeichnungen der Messstellen Hummelshain und Jägersdorf und die Abflussmessungen der bedeutenden Vorfluter im Einzugsgebiet ausgewertet. Unter der Annahme, dass der überwiegende Teil des Grundwasserabflusses im Buntsandstein über das Kluftsystem erfolgt, errechnet sich aus Gleichung 4 ein mittlerer Speicherkoeffizient von ca. 3\%. Diese Größenor- dung konnte durch Pumpversuche im Untersuchungsgebiet und durch vergleichende Angaben für Kluftsysteme in anderen Buntsandsteingebieten (Eissele 1966, Udluft 1971, 1972, Schubuth 1973, März 1977, Georgotas \& Udluft 1978 und Huber 1992) bestätigt werden.

Basierend auf dem ermittelten Speicherkoeffizienten von $3 \%$ für das Kluftsystem wurde im Zeitraum 1999 bis 2001 der schnelle Grundwasserneubildungsanteil nach 
Abb. 8 Bestmöglichste Korrelation zwischen geglätteter Niederschlagsganglinie der Station Hummelshain und den langfristigen Schwankungen des Grundwasserganges in der Messstelle Hummelshain

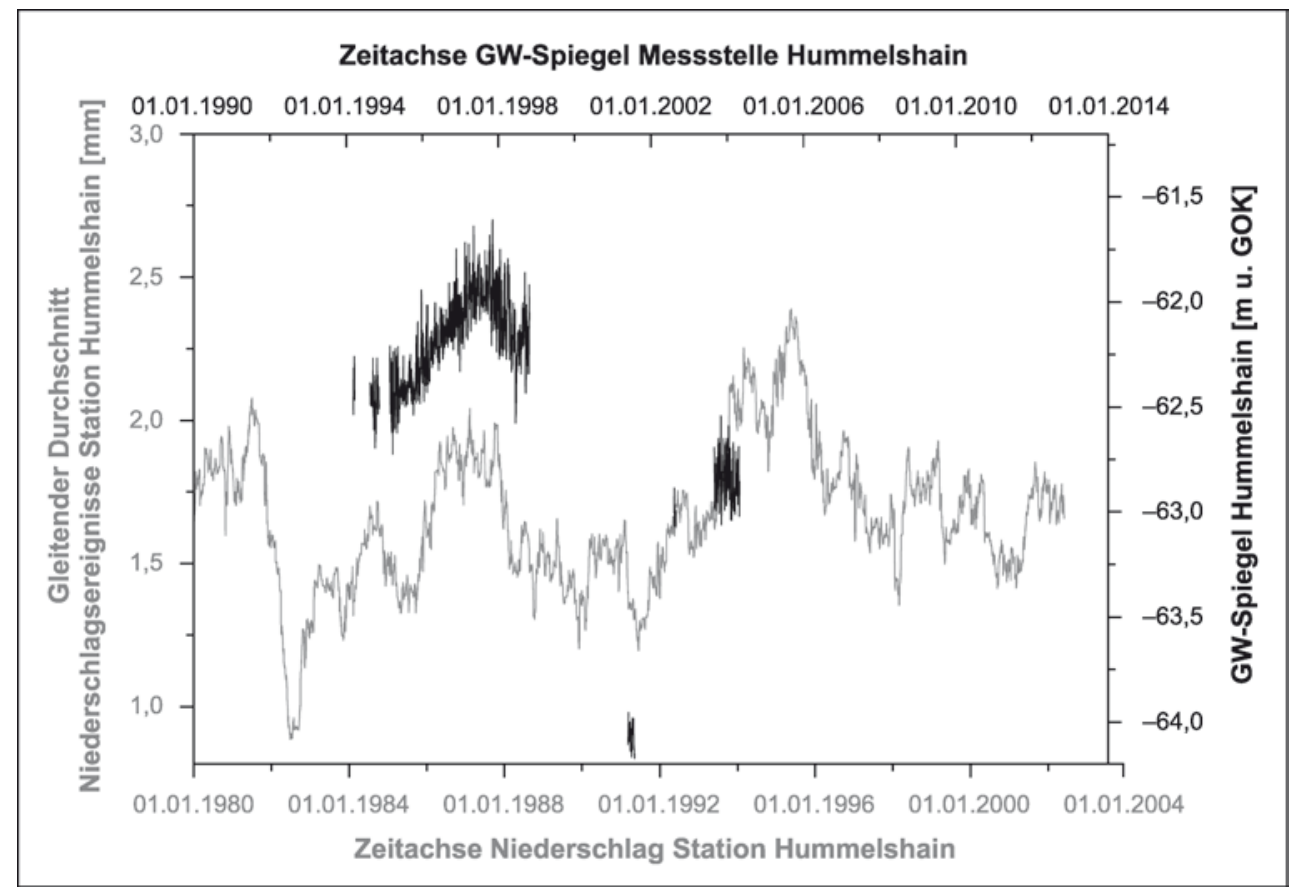

der WTF-Methode berechnet. Im Anschluss wurden diese Ergebnisse denen einer Bodenwasserbilanzberechnung (Sauter 1992) gegenübergestellt. Durch den Vergleich der jeweiligen Jahressummen für die Grundwasserneubildung von 1999 bis 2001 konnte der verbleibende langsame Grundwasserneubildungsanteil für das Porensystem ermittelt werden. Durch die Kombination der WTF-Methode mit einem Bodenwasserhaushaltsmodell konnte für das Porensystem ein Speicherkoeffizient von ca. $5 \%$ bestimmt werden, der mit Literaturangaben für Speicherkoeffizienten in ungespannten Aquiferen gut übereinstimmt. Aus den ermittelten Speicherkoeffizienten für das Kluft- und Porensystem ergibt sich, dass ca. 70\% der Grundwasserneubildung über das Großkluftsystem und ca. $30 \%$ über die feingeklüftete Matrix infiltrieren. Ähnliche Größenordnungen leitete auch Matthess (1970) für den Anteil der Porenwasserbewegung im nordhessischen Buntsandsteinbecken $a b$.

Plausibilitätskontrolle der Korrelationsergebnisse

Die Ergebnisse der statistischen Korrelationsverfahren und der WTF-Methode wurden mit den berechneten Grundwasserneubildungsdaten aus dem Bodenwasserhaushaltsmodell (Sauter 1992) verknüpft. Unter Beachtung der zeitlichen Verzögerung der dualen Fließsysteme und deren Volumenprozente wurden Grundwasserneubildungsfunktionen in Abhängigkeit des Flurabstandes generiert, die für die instationäre numerische Modellierung mittels VISUAL MODFLOW 3.0 notwendig war.
Das zunächst erstellte stationäre Modell wurde an zwei Grundwassergleichenplänen mit repräsentativen Wasserständen, deren Grundlage Stichtagsmessungen im Oktober 2002 und April 2003 waren, kalibriert. Die Validierung des instationären Strömungsmodells erfolgte durch die aufgezeichneten Grundwasserganglinien. Eine stellenweise Nachkalibrierung des Modells erfolgte durch die Anpassung der Durchlässigkeitsbeiwerte und der Speicherkoeffizienten. Mit den Modellergebnissen der stationären und instationären Strömungsmodellierung wurden die analysierten zeitlichen Verschiebungen zwischen Niederschlagsereignissen und den zugehörigen Grundwasserspiegelschwankungen an den verschiedenen Messstellen nachgebildet (Abb. 9). Für die Messstellen Jägersdorf und Hummelshain ergaben sich die besten Anpassungen zwischen simulierten und gemessenen kurzfristigen Grundwasserspiegelschwankungen bei einer zeitlichen Verzögerung der Niederschlagsereignisse von 7 Tagen. Dagegen wurden die gemessenen kurzfristigen Grundwasserspiegelschwankungen in der Messstelle Schleifreisen am besten mit einer 4-tägigen Verzögerung der Niederschlagsereignisse nachgebildet. Die langfristigen Reaktionen auf Niederschlagsereignisse wurden für Schleifreisen mit ca. 5 Jahren, für Jägersdorf mit $51 / 2$ Jahren und für Hummelshain mit 10 Jahren simuliert. Die Ergebnisse der instationären Modellierung in Abb. 9 bestätigten damit die Ergebnisse aus den Kreuzkorrelationen und die quantifizierten Grundwasserneubildungsanteile des Kluft- und Porensystems. Um eine eindeutiges Aussage für die Messstellen Hummelshain und Schleifreisen zu treffen, sollte jedoch ein längerer Messzeitraum des Grundwassergangs vorliegen. 
Abb. 9 Vergleich zwischen simulierten (grau) und gemessenen (schwarz) Grundwasserständen

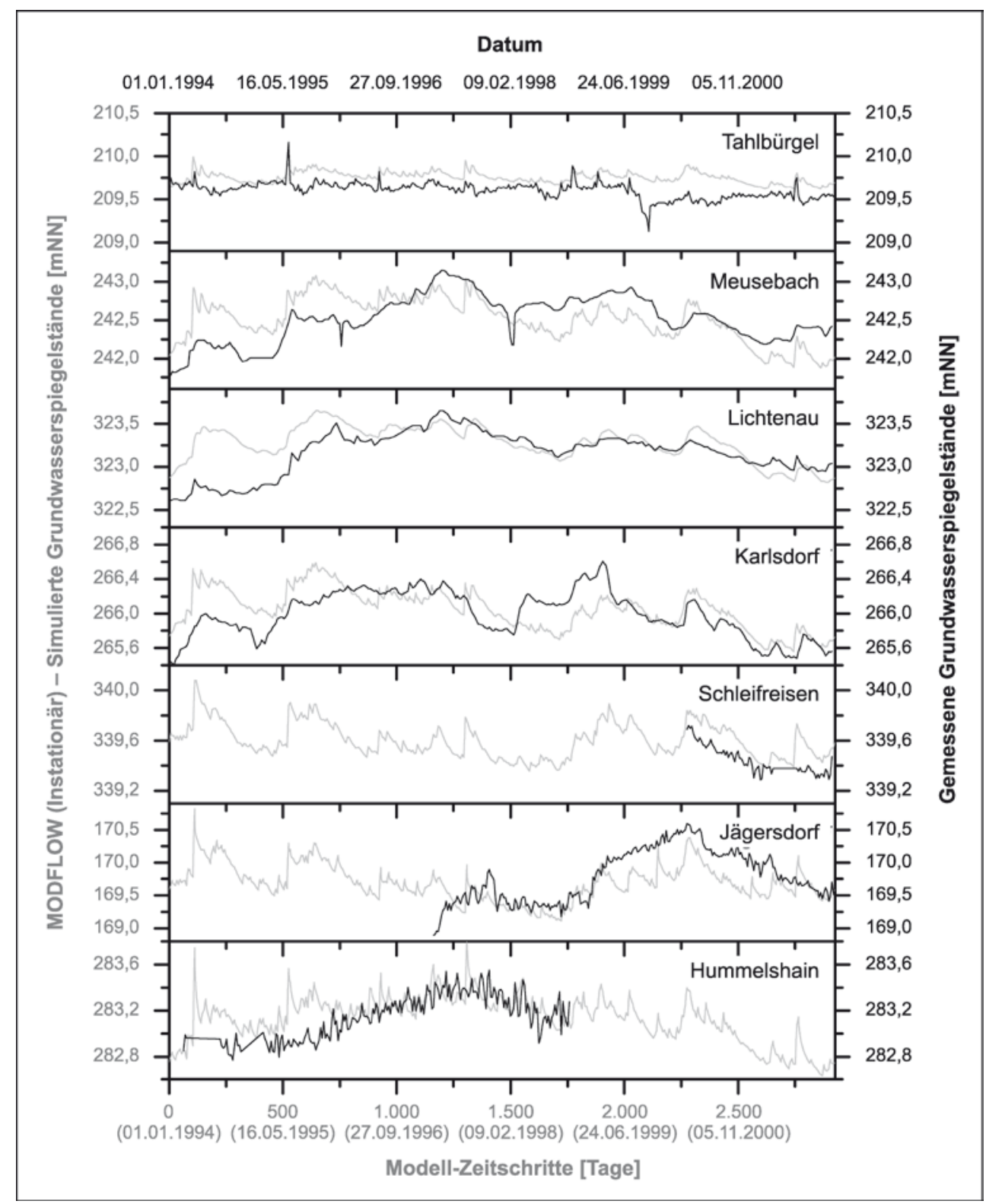

\section{Zusammenfassung}

Analysen des Grundwassergangs im Buntsandsteingrundwasserleiter Ostthüringens zeigen eine deutliche Trennung der Grundwasserneubildung in zwei Komponenten. Über Großklüfte erfolgt eine schnelle Infiltration und über die feingeklüftete Sandsteinmatrix infiltriert eine deutlich verzögerte zweite Komponente. Die Infiltrationszeiten betragen 2-14 Tage für die schnelle und 5-10 Jahre für die langsame Komponente, wobei die Variabilität sowohl vom Flurabstand als auch von Unterschieden im kluftdominierten und matrixdominierten Strömungsregime abhängt. Durch die Verzögerung des Grundwasserneubildungsanteils des matrixdominierten Strömungsregimes kann sich ein großes ungenutztes Wasserreservoir ansammeln. Dieses zeigt sich in den langfristigen Wasserspiegelschwankungen mit Amplituden von einigen Dezimetern bis maximal 1 Meter. Durch die Kombination der WTF-Methode mit einem Bodenwasserhaushaltsmodell konnten die Grundwasserneubildungsanteile für das Kluftsystem mit ca. 70\% und für das Porensystem mit ca. $30 \%$ quantifiziert werden.

Open Access Dieser Artikel wird zu den Bedingungen der „Creative Commons Attribution Noncommercial License" zur Verfügung gestellt. Damit ist eine nichtkommerzielle Nutzung, Verbreitung und Vervielfältigung erlaubt, sofern die Autoren des Artikels und die genaue Quelle angegeben sind. 


\section{Literatur}

Davis, J. C.: Statistic and data analysis in geology.- 564 S.; Wiley, New York (1986)

Dürbaum, H. J., Matthess, G., Rambow, D.: Untersuchungen der Gesteins- und Gebirgsdurchlässigkeit des Buntsandsteins in Nordhessen.- Notizbl. hess. Landesamtes Bodenforsch. 97, 258-274 (1969)

Einsele, G., Merklein, J.: Aquiferdaten von Fest- und Lockergesteinen im Gebiet des Mittelmains und der Regnitz.- Schriftr. Bayer. Landesamt für Wasserwirtschaft 7, 240-250 (1978)

Eissele, K.: Über die Grundwasserbewegung in klüftigen Buntsandstein.- Jh. Geol. L.-Amt Baden-Württemberg 8, 105-111 (1966)

Georgotas, N., Udluft, P.: Sinn und Fränkische Saale.- Schriftr. Bayer. Landesamt für Wasserwirtschaft 7, 65-84 (1978)

Hauthal, U.: Zum Wasserleitvermögen von Gesteinen des Mittleren Buntsandsteins. Zeitschrift für angewandte Geologie 13(8), 405407 (1967)

Harzer, E: Grundwasserneubildungsdynamik im Buntsandstein des Spessarts - Ermittlung über die Durchströmung der ungesättigten Zone.- Diss. Univ. Würzburg. (2003)

Healy, R. W., Cook, P. G.: Using groundwater levels to estimate recharge.- Hydrogeol. Journal 10, 91-109 (2002)

Hecht, G.: Grundwässer.- In Seidel, G.: Geologie von Thüringen.556 S.; Schweizerbart, Stuttgart (1995)

Hölting, B.: Hydrogeologie.- 415 S.; Enke, Stuttgart (1992)

Huber, B.: Der Einfluß des Trennflächengefüges auf die Grundwasserströmung in Kluftgrundwasserleitern.- Hydrogeologie und Umwelt 5, 293 (1992)

Matthess, G.: Beziehung zwischen Bau und Grundwasserbewegung in Festgesteinen.- 105 S., Abh. Hess. L.-Amt f. Bodenforsch. 58 (1970)
März, K.: Hydrogeologische und hydrochemische Untersuchungen im Buntsandstein und Muschelkalk Nordbayerns.- Hydrochem. Hydrogeol. Mitt. 2, 1-170 (1977)

Merkel, B.J., Planer-Friedrich, B.: Integrierte Datenauswertung Hydrogeologie.- Freiberger Online Geology (FOG) 7 (2002)

Rödiger, T.: Charakterisierung und Modellierung des Buntsandsteinfließsystems im Osten des Thüringer Beckens.- Diss. Univ. Jena (2005)

Sauter, M.: Quantification and forecasting of regional groundwater flow and transport in a karst aquifer (Gallusquelle, Malm, SW Germany).- Tüb. Geow. Arb. (TGA) C13.- 150 S.; Tübingen. (1992)

Schraft, A., Rambow, D.: Vergleichende Untersuchungen zur Gebirgsdurchlässigkeit im Buntsandstein Osthessens.- Geol. Jb. Hessen 112, 235-261 (1984)

Schubuth, H.: Beziehung zwischen Grundwasserabfluss und tektonischen Bau im Buntsandstein des Mainvierecks zwischen Lohr und Aschaffenburg im Spessart.- Diss. Univ. Frankfurt a.M. (1973)

Sophocleous, M.A.: Combining the soilwater balance and waterlevel fluctuation methods to estimate natural groundwater recharge: practical aspects.- Journal of Hydrology 124, 229-241 (1991)

Udluft, P.: Hydrogeologie des Oberen Sinntales; ein Beitrag zur Kenntnis der bayerischen Rhön.- Geolica Bavarica 64, 365-384 (1971)

Udluft, P.: Bestimmung des entwässerbaren Kluftraumes mithilfe des Austrocknungskoeffizienten nach Maillet, dargestellt am Einzugsgebiet der Lohr (Nordost Spessart).- Z. dt. geol. Ges. 123, 53-63 (1972)

WHI: Visual MODFLOW v.3.0 User's Manual. 370 S.; Waterloo Hydrogeologic Inc. (2002) 